\title{
Effects of Potato-Psyllid-Vectored 'Candidatus Liberibacter solanacearum' Infection on Potato Leaf and Stem Physiology
}

\author{
C. M. Wallis, A. Rashed, J. Chen, L. Paetzold, F. Workneh, and C. M. Rush
}

First and third authors: United States Department of Agriculture-Agricultural Research Service, San Joaquin Valley Agricultural Sciences Center, Crop Diseases, Pests and Genetics Research Unit, 9611 S. Riverbend Ave, Parlier, CA 93648; second author: Department of Plant, Soil, and Entomological Sciences, University of Idaho Aberdeen Research and Extension Center, Aberdeen 83210; and fourth, fifth, and sixth authors: Texas A\&M AgriLife Research, 6500 Amarillo Blvd. W., Amarillo 79106.

Accepted for publication 23 August 2014.

\begin{abstract}
Wallis, C. M., Rashed, A., Chen, J., Paetzold, L., Workneh, F., and Rush, C. M. 2015. Effects of potato-psyllid-vectored 'Candidatus Liberibacter solanacearum' infection on potato leaf and stem physiology. Phytopathology 105:189-198.

The bacterium 'Candidatus Liberibacter solanacearum' is associated with zebra chip disease (ZC), a threat to potato production in North America and New Zealand. It is vectored by potato psyllids. Previous studies observed that ' $\mathrm{Ca}$. L. solanacearum' infection causes potato tubers to undergo ZC-symptom-associated shifts in physiology, such as increased levels of amino acids, sugars, and phenolics. However, little is known about how ' $C a$. L. solanacearum' infections caused by psyllid vector

positive psyllids with potato plants not exposed to psyllids. Foliar levels of asparagine, aspartic acid, glutamine, fructose, glucose, sucrose, a ferulic acid derivative, and quinic acid were lower in ' $\mathrm{Ca}$. L. solanacearum'inoculated than noninfected plants. However, foliar levels of proline, serine, four phenolic compounds, and most terpenoids were greater in ' $C a$. L. solanacearum'-inoculated than noninfected plants. Upper stem levels of asparagine and aspartic acid, upper and lower stem levels of ellagitannins and most monoterpenoids, and lower stem level of sesquiterpenoids were greater in 'Ca. L. solanacearum'-inoculated than noninfected plants. These results suggest that many defense-related terpenoid compounds might increase in plants which had psyllids inoculate ' $\mathrm{Ca}$. L. solanacearum'. This could impact progression and spread of ZC.
\end{abstract} feeding may affect metabolism in potato foliage and stems. This study compared metabolism in potato plants fed upon by ' $\mathrm{Ca}$. L. solanacearum'-
Additional keyword: carbohydrates.
Potato (Solanum tuberosum L.) production in Central America, western North America, and New Zealand is threatened by zebra chip disease (ZC), putatively caused by 'Candidatus Liberibacter solanacearum' (syn. 'Ca. L. psyllaurous') (10,11,21,29,35,46). Other potato-growing regions could have $\mathrm{ZC}$ spread to them as well (18). Effective ZC management currently involves costly $(\approx \$ 700 /$ ha $)$ chemical control programs for the potato psyllid vector, Bactericera cockerelli (Sulc) (16). Long-term management would need to exploit natural host resistance, including discovering resistance markers for reduced symptom development when plants are infected with ' $C a$. L. solanacearum' or potato plants where vectoring efficiency is reduced $(27,28)$.

Characteristic ZC symptoms include increased oxidation (browning) of freshly cut tubers and brown striping ("zebra stripes") when tuber slices are fried $(27,36,46)$. ZC also causes symptoms in aerial tissues of potato plants, including plant dwarfing, formation of aerial tubers, leaf scorch, chlorosis, and plant collapse $(27,36,46)$.

Tuber ZC symptoms are associated with increased levels of phenolics $(1,30,33,41,44)$, reducing sugars (glucose and fructose) $(7,8,15,33,44)$, defense-associated enzymes $(1,33,41)$, amino acids (e.g., isoleucine, leucine, serine, tyrosine, and valine) $(30,33,41$, 44), and minerals (e.g., phosphorus, potassium, and calcium) (24).

All of these physiological changes could be attributed to host attempts to combat infection via a hypersensitive response (25). In particular, increased levels of phenolics and terpenoids are

Corresponding author: C. M. Wallis; E-mail address: christopher.wallis@ars.usda.gov

http://dx.doi.org/10.1094/PHYTO-04-14-0113-R

This article is in the public domain and not copyrightable. It may be freely reprinted with customary crediting of the source. The American Phytopathological Society, 2015. often associated with hypersensitive responses and other general coordinated host responses to pathogens $(5,17,31,42)$. Phenolic compounds are precursors to the cell-wall-thickening lignin and tannins, which could make host tissues harder to penetrate by mircoorganisms $(42,43)$. Likewise, both phenolics and terpenoids have putative antibiotic effects against pathogens $(19,20)$. Changes in levels of other compounds may also occur as part of a host defense response. For instance, certain amino acids might become precursors to particular antibiotic or antiherbivory compounds $(6,26)$. However, in the case of ' $\mathrm{Ca}$. L. solanacearum', it remains unclear whether or not the bacterium is negatively impacted by host physiological changes, although some evidence exists that it is impacted somewhat because lower titers may be present where host physiological changes are greatest $(34,41,44)$.

Regardless, it is hypothesized that changes in host physiology result directly in observed ZC symptoms (41). Browning of freshly cut potato tubers would be expected to increase with greater phenolic and polyphenol oxidase levels, because polyphenol oxidases utilize chlorogenic acid and similar phenolics to form brown-colored products upon exposure to air $(23,41)$. Greater browning of fried potato tuber slices would be expected with increased sugar and amino acid levels in tubers, because these compounds cross-link to form a brown acrylamide product at frying temperatures via the Maillard reaction $(14,39,41)$.

However, what physiological changes may occur in leaves and stems of ' $C a$. L. solanacearum'-infected potato plants remains unclear. Physiological shifts could be hypothesized to be associated with the aerial symptoms of $\mathrm{ZC}$ such as dwarfing, aerial tuber formation, chlorosis, leaf scorching, and plant collapse $(30,36)$. Because ' $C a$. L. solanacearum' is vectored by potato psyllids, the combination of host responses to potato psyllid vectors as well as host responses to ' $\mathrm{Ca}$. L. solanacearum' itself 
could result in these symptoms. It has been shown previously that the combination of ' $\mathrm{Ca}$. L. solanacearum' infection and psyllid feeding together inflicted much greater symptom formation on potato hosts, and likely changed physiology greater, than when psyllid feeding occurred alone (37). In terms of previous studies examining shifts in host physiology associated with ZC, preliminary work focused on examining foliar sugar and starch content from plants fed upon by psyllids that were likely ' $\mathrm{C} a$. L. solanacearum' positive (15). Leaves from psyllid-infested potato plants had lower sugar and greater starch levels than noninfested controls (15). Furthermore, plants with previous ' $C a$. L. solanacearum'-positive potato psyllid infestations had greater terpenoid levels than those that were previously noninfested (13).

Expanded knowledge about the physiological changes that potato leaves and stems undergo when infected with ' $C a$. L. solanacearum' via psyllid inoculation could aid in the development of effective ZC management strategies. For instance, if there is reduced amino acid and sugar production as a result of ' $\mathrm{Ca}$. L. solanacearum' infection, then fewer and smaller tubers would develop in $\mathrm{ZC}$ diseased plants. This is important if management strategies reduce the expression of $\mathrm{ZC}$-associated browning in tubers but fail to address the effects of ' $\mathrm{Ca}$. L. solanacearum' infection and potato psyllid feeding on aerial portions of the host. Furthermore, increased understanding of the host-pathogen interactions of potato infected with ' $\mathrm{Ca}$. L. solanacearum' could be useful in development of ' $\mathrm{Ca}$. L. solanacearum' resistance or ZC tolerance markers for potato breeding programs.

In addition to potential effects on ' $\mathrm{Ca}$. L. solanacearum' success, infection-induced changes in potato host leaf or stem physiology due to ' $C a$. L. solanacearum' infections could manipulate potato host attractiveness to additional feeding by potato psyllids. This, in turn, could affect the likelihood of ' $\mathrm{Ca}$. L. solanacearum' spread beyond initially infected plants. Often, plants may respond to pathogen infections and insect infestations by producing greater levels of secondary metabolites such as certain phenolics and terpenoids, both of which could influence insect behaviors (42). Davis et al. (13) observed that potato psyllids were more likely to settle on previously ' $C a$. L. solanacearum'-positive psyllid-infested potato plants than those that were not previously infested, although this effect became nonsignificant later. This observation might be the result of increased terpenoid levels that occurred in psyllid-infested host plants (13). Alternatively, differences in psyllid preference may have been the result of other, nonexamined changes in leaf and stem physiology.

This study examined levels of both primary (amino acids and carbohydrates) and secondary (phenolics and terpenoids) metabolites in healthy potato plants ('Russet Norkotah') and those infected with ' $C a$. L. solanacearum' by potato psyllid vectors. This was done to address the hypothesis that ' $\mathrm{Ca}$. L. solanacearum' infections started by psyllid vectors significantly alter potato leaf and stem physiology. Furthermore, relationships were determined between levels of these host compounds with ZC symptoms and ' $C a$. L. solanacearum' titers. Knowledge gained from this study is useful in understanding the effects that ' $\mathrm{Ca}$. L. solanacearum' infection has on ZC symptom progression of aerial parts of the potato host, with implications for ZC management.

\section{MATERIALS AND METHODS}

Experimental design. Russet Norkotah seed potato plants were planted on 19 April 2012 at Texas A\&M AgriLife Research and Extension Center in Bushland, TX, and covered with tentshaped mesh cages $(100$ by 100 by $100 \mathrm{~cm})$ before plant emergence. In May, plant thinning was conducted to reduce the number of plants to four in each cage. Treatments were assigned to the plants in a randomized, complete block design.

Plants were infected with ' $\mathrm{Ca}$. L. solanacearum' according to the methods of Wallis et al. (44). In brief, potato psyllids of a central haplotype (40), randomly selected from a ' $\mathrm{Ca}$. L. solanacearum'-positive greenhouse colony, were used to infest 16 potato plants ( 4 plants in each of four replicate cages). To confirm the 'Ca. L. solanacearum'-positive status of the colony, three composite samples of 10 psyllids were tested prior to our experiments. In addition, the greenhouse colony was tested for other inoculation studies and on a weekly basis. It never tested negative for ' $\mathrm{Ca}$. L. solanacearum'. The biotype of ' $\mathrm{Ca}$. L. solanacearum' in the potato psyllid colony was determined to be a mix of ' $\mathrm{Ca}$. $\mathrm{L}$. solanacearum' A and B (45). ' $C a$. L. solanacearum' inoculations involved releasing 30 psyllids from the ' $\mathrm{Ca}$. L. solanacearum'positive colony at the base of a single plant in each $100-\mathrm{cm}^{3}$ cage containing four plants 4 weeks prior to harvest. Another 16 potato plants were left noninfested as controls (4 plants in each of four replicate cages). After 1 week, both infested and noninfested plants were thoroughly sprayed with a mix of the insecticides Movento (Bayer CropScience), Agri-Mek (Syngenta Crop Protection, LLC), and Fulfill (Syngenta) to remove the potato psyllids. The pesticide mix was reapplied a week later to both infested and noninfested plants to assure insect removal.

Plants were harvested on 26 July 2012. Plants were cut at soil level, bagged, labeled, and placed immediately on dry ice. These plants were then shipped overnight on dry ice to San Joaquin Valley Agricultural Sciences Center, United States Department of Agriculture-Agricultural Research Service, in Parlier, CA. Overall aerial ZC symptoms were assessed on a scale of 0 to 3, where $0=$ healthy; 1 = lightly symptomatic, less than $25 \%$ discolored tissue; $2=$ moderately symptomatic, 25 to $75 \%$ discolored tissue; and $3=$ severely infected, greater than $75 \%$ tissue discoloration. The ratings are equivocal to the 0 -to-4 scale visualized within Rashed et al. (31), with plants rated $4=$ dead not sampled for this study.

'Ca. L. solanacearum' titer and biochemical analyses. Potato leaf and stem samples were stored at $-20^{\circ} \mathrm{C}$ before biochemical analyses. Upon removal from storage, the samples were separated by leaf, upper stem material (within $\approx 10 \mathrm{~cm}$ of the apical end of the plant), and lower stem material (within $\approx 10 \mathrm{~cm}$ of the soil line). Regardless of leaf or stem material, the tissues were pulverized via mortar and pestle in liquid nitrogen. For each sample, three 0.10 -g aliquots were weighed out and placed into separate, labeled $1.5-\mathrm{ml}$ microcentrifuge tubes, and flash-frozen in liquid nitrogen to prevent oxidation.

Additional tissue aliquots had DNA extracted using the DNeasy Plant mini kit (Qiagen, Valencia, CA) for titer analyses. The methods of Wallis et al. (41) were used to conduct quantitative polymerase chain reaction (PCR) for the determination of ' $\mathrm{Ca}$. $\mathrm{L}$. solanacearum' titers. In brief, each quantitative PCR involved $2 \mu \mathrm{l}$ of extracted tissue DNA template, $12.5 \mu \mathrm{l}$ of $2 \times$ iQ SYBR Green supermix (Bio-Rad, Hercules, CA), $0.5 \mu \mathrm{l}$ of both $10 \mu \mathrm{M}$ OA2 and $10 \mu \mathrm{M}$ OI2c primers (21), and $9.5 \mu \mathrm{l}$ of water for a total reaction solution of $25 \mu \mathrm{l}$ (41). A DNA Engine Opticon 2 Real Time PCR Detection System (Bio-Rad) was used to monitor the reactions, Cycle threshold values were calculated by the system software, and standard curves of known DNA amounts were performed to tabulate ' $C a$. L. solanacearum' titers of each sample (41).

The methods of Wallis et al. (42), Rashed et al. (33), and Wallis et al. (44) were employed to analyze plant biochemistry. In brief, the $0.10-\mathrm{g}$ aliquots were twice-extracted overnight at $4^{\circ} \mathrm{C}$ in $0.5 \mathrm{ml}$ of phosphate-buffered saline (PBS) solution ( $\mathrm{pH} \mathrm{7.8)}$ to analyze amino acids and carbohydrates, $0.5 \mathrm{ml}$ of methanol (Sigma-Aldrich, St. Louis) to analyze phenolic compounds, or $0.5 \mathrm{ml}$ of methyl tert-butyl ether (MTBE) (Sigma-Aldrich) to analyze terpenoids (for a total of $1 \mathrm{ml}$ of each extract).

A total of $100 \mu \mathrm{l}$ of the PBS buffer extract was used to assess amino acids using the commercially available kit from Phenomenex (Torrance, CA). Processed amino acid extracts then were analyzed with a Shimadzu (Columbia, MD) GC-2010 gas chro- 
matograph (GC) with a flame ionization detector (FID) and a Zebron AAA column, with identification and quantification performed using standard preparations from the Phenomenex amino acid kit.

To analyze carbohydrates, $50 \mu \mathrm{l}$ of the PBS buffer extract was injected into a Shimadzu high-performance liquid chromatograph (HPLC) equipped with Supelco C-611 carbohydrate column (300 by $7.8 \mathrm{~mm}$ ) (Sigma-Aldrich) and a refractive index detector (RID10 from Shimadzu) (33). Fructose, glucose, and sucrose commercial standards (all from Sigma-Aldrich) were used to confirm peak identifies and to convert peak areas to gram amounts.

To analyze phenolics, $50 \mu \mathrm{l}$ of methanol extract was injected into a Shimadzu HPLC system equipped with an XR-ODS C18 column (Shimadzu) and a Shimadzu photodiode array detector (set at $280 \mathrm{~nm})(33,44)$. A binary solvent program was used, proceeding for $95 \%$ solvent A (water with $0.2 \%$ [vol/vol] acetic acid) (Sigma-Aldrich) to $100 \%$ solvent B (methanol with $0.2 \%$ [vol/vol] acetic acid) and back to reset for the following injection over $40 \mathrm{~min}$. Most compounds were previously identified by Wallis et al. (44). However, additional phenolic compounds were identified and all compounds quantified into milligrams per gram fresh weight $(\mathrm{FW})$ by matching retention times with commercial standards (from Sigma-Aldrich), matching UV/Vis spectra of peaks, or determining molecular weights by running representative samples on a Shimadzu LCMS-2020 liquid chromatographmass spectrometer with the same binary gradient program. Peaks were converted to gram amounts using compounds within the same compound class when commercial standards were unavailable (33).

To analyze terpenoids and volatile compounds, $1 \mu \mathrm{l}$ of the MTBE extract was injected into a Shimadzu GC-2010 GC system with a $\beta$ Dex column (Restek, Bellefonte, PA) and an FID. At a constant linear velocity for helium of $30 \mathrm{ml} / \mathrm{min}$, the oven program changed from 40 to $210^{\circ} \mathrm{C}$ over $40 \mathrm{~min}$ (with an original hold of $5 \mathrm{~min}$ at $40^{\circ} \mathrm{C}$ ). Commercial standards (all from SigmaAldrich) were used to identify compound peaks and to convert amounts into micrograms per gram FW amounts. For the compounds that did not have commercial standards, identifications were made using a Shimadzu GC system equipped with the same column and a GC-MS2010S mass spectrometer detector running the same GC-FID program. Standards curves from similar, commercially available compounds were used to convert unknowns into nanogram per gram FW amounts, with $\alpha$-pinene used as the external standard for conversion of monoterpenoids and $\beta$ caryophellene used as the external standard for conversion of sesquiterpenoids.

Statistical analyses. All statistics were performed using IBMSPSS software (version 19.0; IBM, Armonk, NY) and $\alpha=0.05$. Normality tests were performed using the SPSS EXPLORE feature. For each compound class (and subclasses for terpenoids and volatiles), multivariate analyses of variance (MANOVA) was utilized to examine differences between noninfected and infected plants for each tissue type. Blocks were originally included but later removed from the final model because they were always nonsignificant $(P>0.05)$. In addition to blocks, the plant number within the cage also was included due to the psyllids being released on only one plant (which was designed as "plant 1 " in all cages). However, plant number was also removed from all tests because it was always nonsignificant $(P>0.05)$. If the MANOVA Pillai's trace statistic was significant, follow-up analyses of variance (ANOVAs) were performed on individual compounds. Spearman's rank correlations were used to determine associations between aerial and tuber ZC symptoms using both noninfected and ' $C a$. L. solanacearum'-infected plants, to determine whether changes in aerial symptoms related to $\mathrm{ZC}$ belowground. Likewise, Spearman's correlations were used to determine correlations between compound levels in infected plants and aerial ZC symptoms and ' $\mathrm{Ca}$. L. solanacearum' titers. Positive correlations indi- cated that greater compound levels were associated with greater symptoms or greater ' $C a$. L. solanacearum' titers.

\section{RESULTS}

Associations between aerial symptoms with tuber symptoms, aerial symptoms, and ' $\boldsymbol{C} \boldsymbol{a}$. L. solanacearum' titers. Symptom ratings for leaves (Spearman $\rho=0.659 ; P<0.001 ; N=$ $29)$, lower stems $(\rho=0.611 ; P<0.001 ; N=32)$, and upper stems $(\rho=0.589 ; P<0.001 ; N=32)$ were positively associated with tuber symptoms.

There were no significant associations between symptoms and 'Ca. L. solanacearum' titers in leaves $(\rho=-0.382 ; P=0.178 ; N=$ $14)$, upper stems $(\rho=-0.243 ; P=0.364 ; N=16)$, or lower stems $(\rho=-0.070 ; P=0.797 ; N=16)$.

' $\mathrm{Ca}$. L. solanacearum' infection effects on levels of amino acids. In total, 17 amino acids (alanine, asparagine, aspartic acid, glutamic acid, glutamine, glycine, histidine, isoleucine, leucine, lysine, methionine, phenylalanine, proline, serine, tryptophan, tyrosine, and valine) were analyzed in both leaf and stem tissue. In leaf tissue, infected plants had significantly different amino acid levels than noninfected plants according to MANOVA (Pillai's trace $\Delta=0.944 ; F=5.631 ; P=0.021 ; N=25$ ). Serine and proline foliar levels were significantly greater in ' $\mathrm{Ca}$. L. solanacearum'-infected than noninfected plants (Table 1). In contrast, noninfected plants had significantly greater foliar levels of asparagine, aspartic acid, and glutamine than ' $\mathrm{Ca}$. L. solanacearum'-infected plants (Table 1). Tyrosine levels were negatively correlated with leaf symptoms (Table 1).

Amino acids in upper stems were significantly different, on average, in ' $C a$. L. solanacearum'-infected plants than noninfected plants, according to MANOVA $(\Delta=0.950 ; F=5.328$; $P=0.037 ; N=24)$. In upper stems, levels of asparagine, aspartic acid, and threonine were significantly greater in ' $\mathrm{Ca}$. L. solanacearum'-infected than noninfected plants (Table 1). Tyrosine levels in upper stem tissue were negatively correlated with upper stem symptoms (Table 1).

There were no significant overall effects of ' $\mathrm{Ca}$. L. solanacearum' infection on lower stem levels of amino acids according to MANOVA ( $\Delta=0.744 ; F=1.455 ; P=0.288 ; N=28)$. However, asparagine, aspartic acid, and leucine levels in lower stem tissue were significantly negatively correlated with lower stem symptoms (Table 1). Asparagine, aspartic acid, glutamine, histidine, and lysine levels in lower stem tissue were significantly positively correlated with lower stem ' $\mathrm{Ca}$. L. solanacearum' titers (Table 1).

' $\boldsymbol{C a}$. L. solanacearum' infection effects on carbohydrates. Fructose, glucose, and sucrose levels were examined in all tissues. In leaf $(\Delta=0.625 ; F=15.582 ; P<0.001 ; N=32)$, upper stem $(\Delta=0.399 ; F=3.324 ; P=0.048 ; N=19)$, and lower stem $(\Delta=$ $0.512 ; F=7.330 ; P=0.002 ; N=25)$ tissues, overall sugar levels were significantly greater in noninfected than ' $\mathrm{Ca}$. L. solanacearum'-infected plants. Levels of fructose, glucose, and sucrose alone were also significantly greater in noninfected plants in all tissue types, with the exception of fructose levels in upper stem tissue (Fig. 1). Fructose levels in leaves were negatively associated with 'C $a$. L. solanacearum' titers $(\rho=-0.532 ; P=0.034 ; N=$ 16). Fructose levels in upper stem tissue were negatively associated with symptoms $(\rho=-0.880 ; P=0.021 ; N=6)$. Sucrose levels in lower stem tissue were positively associated with lower stem symptoms $(\rho=0.661 ; P=0.005 ; N=16)$. No other significant correlations were observed between sugar levels, symptoms, or ' $\mathrm{Ca}$. L. solanacearum' titers.

' $\boldsymbol{C a}$. L. solanacearum' infection effects on phenolics. In total, 22 phenolics were quantified in leaf tissue and 14 phenolics were quantified in stem tissues (Table 2). Overall, noninfected plant phenolics levels were different than ' $\mathrm{Ca}$. L. solanacearum'infected plant levels for leaf $(\Delta=0.993 ; F=54.490 ; P<0.001$; 
$N=32)$, upper stem $(\Delta=0.696 ; F=2.774 ; P=0.024 ; N=32)$, and lower stem $(\Delta=0.999 ; F=936.611 ; P<0.001 ; N=32)$ tissues.

In leaf tissue, ' $\mathrm{Ca}$. L. solanacearum'-infected plants had lower levels of betuloside, diflavone, ferulic acid derivative, quercetin diglycoside 2, and quinic acid than noninfected controls (Table 3 ). In contrast, ' $\mathrm{Ca}$. L. solanacearum'-infected plants had greater levels of dichlorogenic acid, epicatechin gallate, procyanidin A, procyanidin $\mathrm{B}$ isomer 4 , and procyanidin digalloyl dimer than noninfected plants (Table 3 ). In the upper stem, ' $C a$. L. solanacearum'-infected plants had greater levels of a flavonoid rhamnoside glycoside, ellagitannin1, ellagitannin 2, and quinic acid than noninfected plants (Table 3 ). In the lower stem, ' $\mathrm{Ca}$. L. solana- cearum'-infected plants had significantly greater levels of chlorogenic acid, ellagitannin 1, ellagitannin 2, a flavonoid glycoside, and a flavonoid rhamnoside glycoside than noninfected plants (Table 3). However, noninfected plants had greater lower stem levels of a syringic acid derivative than ' $\mathrm{Ca}$. L. solanacearum'infected plants (Table 3 ).

Leaf tissue levels of epicatechin gallate, ferulic acid derivative, flavanol methyl ester, procyanidin B isomer 1, procyanidin B isomer 2, procyanidin $\mathrm{B}$ isomer 3 , and procyanidin $\mathrm{C}$ isomer 1 were positively associated with foliar symptoms (Table 3). Leaf tissue levels of betuloside, diflavone, ferulic acid derivative, and flavonoid glycoside 3 were negatively associated with foliar ' $\mathrm{Ca}$. L. solanacearum' titers (Table 3). Upper stem levels of apigenin

TABLE 1. Mean ( \pm standard error) levels $(\mu \mathrm{mol} / \mathrm{g}$ FW) of amino acids in noninfected and infected leaf, upper stem, and lower stem tissue

\begin{tabular}{|c|c|c|c|c|c|}
\hline Tissue, compound & Noninfected & Infected & $F(N=24-28)$ & Symptom $\rho(N=14-16)$ & Titer $\rho(N=15-16)$ \\
\hline \multicolumn{6}{|l|}{ Leaf } \\
\hline Alanine & $1.37 \pm 0.19$ & $3.36 \pm 0.82$ & 3.819 & 0.398 & 0.094 \\
\hline Asparagine & $5.07 \pm 1.23$ & $2.53 \pm 0.41$ & $5.254 *$ & -0.416 & 0.303 \\
\hline Aspartic acid & $1.78 \pm 0.21$ & $0.599 \pm 0.103$ & $31.658 * * *$ & 0.117 & -0.100 \\
\hline Glutamic acid & $1.12 \pm 0.10$ & $0.673 \pm 0.216$ & 2.544 & -0.046 & -0.229 \\
\hline Glutamine & $3.97 \pm 0.53$ & $1.86 \pm 0.30$ & $13.888 * * *$ & -0.268 & 0.021 \\
\hline Glycine & $0.198 \pm 0.05$ & $0.391 \pm 0.072$ & 3.814 & -0.089 & -0.150 \\
\hline Histidine & $0.340 \pm 0.045$ & $0.356 \pm 0.035$ & 0.083 & 0.064 & 0.297 \\
\hline Isoleucine & $0.854 \pm 0.099$ & $1.00 \pm 0.10$ & 0.979 & -0.105 & 0.009 \\
\hline Leucine & $0.769 \pm 0.096$ & $0.786 \pm 0.072$ & 0.022 & 0.025 & 0.100 \\
\hline Lysine & $0.708 \pm 0.83$ & $0.568 \pm 0.050$ & 2.362 & 0.041 & 0.085 \\
\hline Methionine & $2.17 \pm 0.36$ & $1.43 \pm 0.46$ & 1.351 & 0.105 & -0.100 \\
\hline Phenylalanine & $0.787 \pm 0.097$ & $0.762 \pm 0.070$ & 0.044 & -0.364 & 0.279 \\
\hline Proline & $0.688 \pm 0.130$ & $1.83 \pm 0.42$ & $4.725^{*}$ & -0.311 & -0.085 \\
\hline Serine & $7.58 \pm 0.76$ & $14.9 \pm 2.0$ & $8.352 * *$ & 0.117 & -0.015 \\
\hline Threonine & $0.189 \pm 0.028$ & $0.299 \pm 0.063$ & 1.846 & -0.078 & 0.035 \\
\hline Tryptophan & $0.362 \pm 0.024$ & $0.431 \pm 0.035$ & 2.128 & -0.380 & 0.129 \\
\hline Tyrosine & $0.218 \pm 0.051$ & $0.249 \pm 0.048$ & 0.186 & $-0.542 *$ & 0.285 \\
\hline Valine & $1.79 \pm 0.25$ & $1.80 \pm 0.16$ & 0.001 & 0.048 & 0.062 \\
\hline \multicolumn{6}{|l|}{ Upper stem } \\
\hline Alanine & $2.24 \pm 0.45$ & $4.42 \pm 1.28$ & 2.932 & 0.116 & 0.041 \\
\hline Asparagine & $2.61 \pm 0.59$ & $6.00 \pm 1.44$ & $5.289 *$ & -0.109 & 0.194 \\
\hline Aspartic acid & $0.449 \pm 0.127$ & $1.16 \pm 0.203$ & $9.300 * *$ & -0.188 & 0.136 \\
\hline Glutamic acid & $0.740 \pm 0.116$ & $0.841 \pm 0.110$ & 0.384 & -0.105 & 0.326 \\
\hline Glutamine & $1.53 \pm 0.48$ & $2.32 \pm 0.45$ & 1.405 & -0.123 & 0.325 \\
\hline Glycine & $0.221 \pm 0.046$ & $0.214 \pm 0.054$ & 0.009 & 0.398 & 0.076 \\
\hline Histidine & $0.286 \pm 0.074$ & $0.374 \pm 0.078$ & 0.659 & -0.259 & 0.215 \\
\hline Isoleucine & $0.945 \pm 0.207$ & $1.31 \pm 0.28$ & 1.127 & -0.248 & 0.221 \\
\hline Leucine & $0.643 \pm 0.080$ & $0.715 \pm 0.123$ & 0.257 & -0.295 & 0.194 \\
\hline Lysine & $0.376 \pm 0.075$ & $0.511 \pm 0.090$ & 1.366 & -0.342 & 0.268 \\
\hline Methionine & $0.766 \pm 0.246$ & $0.584 \pm 0.132$ & 0.380 & 0.252 & 0.144 \\
\hline Phenylalanine & $0.427 \pm 0.059$ & $0.540 \pm 0.113$ & 0.847 & -0.266 & 0.059 \\
\hline Proline & $3.24 \pm 2.19$ & $3.48 \pm 1.14$ & 0.008 & -0.052 & 0.297 \\
\hline Serine & $5.96 \pm 0.72$ & $8.46 \pm 2.02$ & 1.544 & 0.103 & 0.012 \\
\hline Threonine & $0.291 \pm 0.036$ & $0.576 \pm 0.133$ & $4.980 *$ & 0.272 & -0.268 \\
\hline Tryptophan & $0.159 \pm 0.039$ & $0.167 \pm 0.030$ & 0.023 & -0.395 & 0.315 \\
\hline Tyrosine & $0.205 \pm 0.045$ & $0.387 \pm 0.126$ & 2.110 & $-0.570 *$ & 0.011 \\
\hline Valine & $1.73 \pm 0.39$ & $2.30 \pm 0.55$ & 0.749 & -0.113 & 0.185 \\
\hline \multicolumn{6}{|l|}{ Lower stem } \\
\hline Alanine & $3.21 \pm 0.66$ & $4.35 \pm 0.86$ & 1.065 & 0.166 & 0.059 \\
\hline Asparagine & $2.92 \pm 0.69$ & $7.04 \pm 1.77$ & 4.195 & $-0.514^{*}$ & $0.624 * *$ \\
\hline Aspartic acid & $1.29 \pm 0.29$ & $1.88 \pm 1.67$ & 1.196 & $-0.529 *$ & $0.568 *$ \\
\hline Glutamic acid & $0.850 \pm 0.194$ & $0.897 \pm 0.194$ & 0.030 & -0.205 & 0.441 \\
\hline Glutamine & $3.36 \pm 0.831$ & $4.35 \pm 0.970$ & 0.575 & -0.456 & $0.564 *$ \\
\hline Glycine & $0.125 \pm 0.035$ & $0.205 \pm 0.042$ & 2.049 & 0.026 & 0.315 \\
\hline Histidine & $0.359 \pm 0.060$ & $0.449 \pm 0.073$ & 0.878 & -0.418 & $0.535^{*}$ \\
\hline Isoleucine & $1.14 \pm 0.22$ & $1.64 \pm 0.33$ & 1.545 & -0.379 & 0.382 \\
\hline Leucine & $0.721 \pm 0.087$ & $0.805 \pm 0.140$ & 0.237 & $-0.529 *$ & 0.353 \\
\hline Lysine & $0.413 \pm 0.069$ & $0.494 \pm 0.085$ & 0.534 & -0.412 & $0.526^{*}$ \\
\hline Methionine & $0.667 \pm 0.317$ & $0.529 \pm 0.138$ & 0.175 & -0.210 & 0.471 \\
\hline Phenylalanine & $0.391 \pm 0.057$ & $0.473 \pm 0.087$ & 0.579 & -0.374 & 0.485 \\
\hline Proline & $2.22 \pm 1.35$ & $5.67 \pm 1.62$ & 2.571 & -0.169 & 0.418 \\
\hline Serine & $6.27 \pm 0.80$ & $9.01 \pm 1.59$ & 2.163 & 0.044 & 0.006 \\
\hline Threonine & $0.563 \pm 0.107$ & $0.724 \pm 0.160$ & 0.654 & -0.202 & -0.065 \\
\hline Tryptophan & $0.144 \pm 0.030$ & $0.169 \pm 0.024$ & 0.428 & -0.453 & 0.368 \\
\hline Tyrosine & $0.193 \pm 0.046$ & $0.275 \pm 0.051$ & 1.357 & -0.383 & 0.274 \\
\hline Valine & $1.99 \pm 0.33$ & $2.97 \pm 0.59$ & 1.939 & -0.324 & 0.376 \\
\hline
\end{tabular}

${ }^{a} F$ test and Spearman's $\rho$ correlation statistics are given; *, **, and $* * *$ indicate $P<0.05,0.01$, and 0.001 , respectively. 
glucoside, chlorogenic acid, and rutin were positively associated with upper stem symptoms (Table 3). Lower stem levels of chichoric acid, chlorogenic acid, and cryptochlorogenic acid ( $\rho=$ 0.546; $P=0.029 ; N=16$ ) were positively associated with lower stem symptoms (Table 3 ). Lower stem levels of a flavonoid rhamnoside glycoside and quinic acid were positively associated with ' $\mathrm{Ca}$. L. solanacearum' titers (Table 3). No other significant correlations were observed between phenolic levels, symptoms, or 'Ca. L. solanacearum' titers.

' $\boldsymbol{C a}$. L. solanacearum' infection effects on terpenoid levels. In total, 17 monoterpenoids (Table 4) and 14 sesquiterpenoids (Table 5) were quantified in potato tissues.

Leaf levels of monoterpenoids $(\Delta=0.912 ; F=6.104 ; P=$ $0.003 ; N=28)$ and sesquiterpenoids $(\Delta=0.736 ; F=2.595 ; P=$ $0.047 ; N=28$ ) were greater in ' $C a$. L. solanacearum'-infected plants than those noninfected. Most individual monterpenoids, with the exception of the two $\beta$-pinene enantiomers, $\alpha$-terpinolene, and allo-ocimene (Table 4), and most sesquiterpenoids, with the exception of $\beta$-gurjunene, $\beta$-farnesene, and $\Delta$-cadinene (Table 5 ), were greater in ' $C a$. L. solanacearum'-infected plants than noninfected plants. There were no significant associations between leaf terpenoid levels with foliar symptoms or ' $\mathrm{Ca}$. L. solanacearum' titers.

Upper stem levels of monoterpenoids were greater in ' $\mathrm{Ca}$. L. solanacearum'-infected plants than those noninfected $(\Delta=0.962$; $F=20.685 ; P<0.001 ; N=32$ ). However, levels of sesquiterpenoids were observed to be greater in noninfected plants than in 'Ca. L. solanacearum'-infected plants $(\Delta=0.902 ; F=11.169$; $P<0.001 ; N=32$ ). With the exceptions of allo-ocimene, bornyl acetate, and terpin-4-ol, individual monoterpenoid levels in the upper stem were greater in ' $\mathrm{Ca}$. L. solanacearum'-infected plants than noninfected plants (Table 4). In contrast, the levels of the sesquiterpenoids $\Delta$-cadinol and germacrene $\mathrm{D}$ were greater in noninfected than ' $C a$. L. solanacearum'-infected plants (Table 5). Upper stem levels of the monoterpenoids $\alpha$-terpinene, $\beta$-linalool, bornyl acetate, terpin-4-ol, and neryl acetate (Table 4), as well as all sesquiterpenoids, except $\beta$-gurjunene (Table 5), were positively correlated with ' $\mathrm{Ca}$. L. solanacearum' titers. No significant associations were observed between upper stem terpenoid levels and symptoms.

Lower stem levels of monoterpenoids $(\Delta=0.949 ; F=14.372$; $P<0.001 ; N=31)$ and sesquiterpenoids $(\Delta=0.785 ; F=4.177$; $P=0.004 ; N=31$ ) overall were greater in ' $C a$. L. solanacearum'infected plants than those noninfected. With the exception of alloocimene and $\beta$-gurjunene, all individual monoterpenoids (Table 4) and sesquiterpenoids (Table 5) were at greater levels in ' $\mathrm{Ca}$. L. solanacearum'-infected plants than noninfected plants. No significant correlations were observed between lower stem levels of monoterpenoids with symptoms or ' $\mathrm{Ca}$. L. solanacearum' titers (Table 4). Lower stem levels of $\beta$-gurjunene, both $\alpha$-farnesene enantiomers, $\alpha$-humulene, $\alpha$-muurolene, $\Delta$-cadinol, and germacrene D-4-ol were negatively associated with ZC symptoms (Table 5).

Lower stem sesquiterpenoid levels were not significantly associated with ' $C a$. L. solanacearum' titers (Table 5).

\section{DISCUSSION}

'Ca. L. solanacearum'-infected plants had significantly different levels of just a few particular amino acids and phenolics compared with noninfected plants in this study, with increases or decreases depending on the compound and tissue type. However, most terpenoid compound levels were generally greater in ' $\mathrm{Ca}$. L. solanacearum'-infected plants than controls, although an ex-

TABLE 2. Putative identifications of phenolic compounds quantified in potato leaf or stem tissues with identification criteria given

\begin{tabular}{|c|c|c|c|}
\hline Tissue, compound & $\begin{array}{l}\text { Retention } \\
\text { time }(\min )^{\mathrm{a}}\end{array}$ & $\begin{array}{l}\text { UV/Vis spectra } \\
\text { maxima }(\mathrm{nm})\end{array}$ & $\begin{array}{c}\text { Molecular } \\
\text { weight }\end{array}$ \\
\hline \multicolumn{4}{|l|}{ Leaf } \\
\hline Betuloside & 27.65 & 279 & 327 \\
\hline Chichoric acid ${ }^{\mathrm{a}}$ & 29.46 & 277 & 473 \\
\hline Dichlorogenic acid & 20.76 & 277 & 705 \\
\hline Diflavone & 25.94 & 277 & 538 \\
\hline Epicatechin gallate $^{\mathrm{a}}$ & 10.92 & 277 & 442 \\
\hline Ferulic acid derivative & 30.26 & 279 & 308 \\
\hline Flavanol methyl ester & 9.79 & 277 & 434 \\
\hline Flavonoid glycoside 1 & 15.17 & 278 & 446 \\
\hline Flavonoid glycoside 2 & 15.35 & 278 & 446 \\
\hline Flavonoid glycoside 3 & 25.67 & 284,310 & 429 \\
\hline Procyanidin A & 15.64 & 280 & 574 \\
\hline Procyanidin B isomer $1^{\mathrm{a}}$ & 13.88 & 278 & 566 \\
\hline Procyanidin B isomer $2^{\mathrm{a}}$ & 14.24 & 278 & 596 \\
\hline Procyanidin B isomer 3 & 18.87 & 278 & 579 \\
\hline Procyanidin B isomer 4 & 20.15 & 278 & 609 \\
\hline Procyanidin $\mathrm{C}$ isomer 1 & 13.70 & 277 & 866 \\
\hline Procyanidin $\mathrm{C}$ isomer 2 & 17.79 & 278 & 866 \\
\hline Procyanidin digalloyl dimer & 17.30 & 275 & 720 \\
\hline Protocatechuic acid hexoside & 6.15 & 260 & 316 \\
\hline Quercetin diglycoside 1 & 16.11 & 277 & 772 \\
\hline Quercetin diglycoside 2 & 17.09 & 275 & 772 \\
\hline Quinic acid ${ }^{\mathrm{a}}$ & 1.62 & 264 & 192 \\
\hline \multicolumn{4}{|l|}{ Stem } \\
\hline Apigenin glucoside & 18.91 & 281,320 & 430 \\
\hline Chichoric acid ${ }^{\mathrm{a}}$ & 29.44 & 264 & 474 \\
\hline Chlorogenic acid ${ }^{\mathrm{a}}$ & 9.16 & 323 & 354 \\
\hline Cryptochlorogenic acid ${ }^{\mathrm{a}}$ & 13.90 & 323 & 354 \\
\hline Ellagitannin 1 & 20.62 & 283,307 & 926 \\
\hline Ellagitannin 2 & 20.80 & 267,313 & 910 \\
\hline Flavonoid diglycoside & 27.41 & 280,314 & 624 \\
\hline Flavonoid glycoside & 15.29 & 280 & 447 \\
\hline Flavonoid rhamnoside glycoside & 20.19 & 273,314 & 579 \\
\hline Protocatechuic acid hexoside & 6.16 & 278 & 316 \\
\hline Quercetin diglycoside & 17.13 & 265,358 & 772 \\
\hline Quinic acid ${ }^{\mathrm{a}}$ & 1.50 & 264 & 192 \\
\hline Rutin $^{\mathrm{a}}$ & 19.65 & 255,347 & 610 \\
\hline Syringic acid derivative & 27.91 & 273,313 & 525 \\
\hline
\end{tabular}

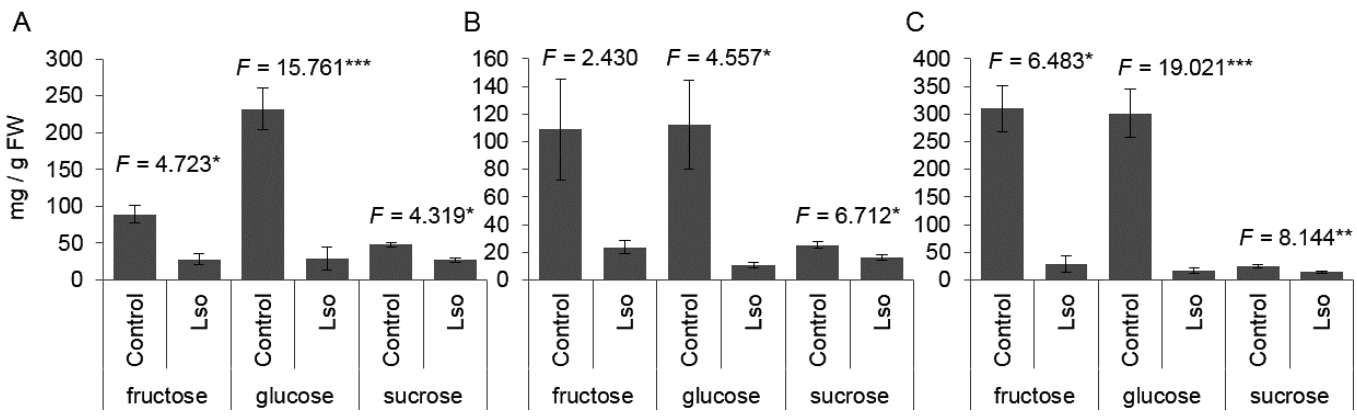

Fig. 1. Mean ( \pm standard error) levels of fructose, glucose, and sucrose in A, leaves; B, upper stems; and $\mathbf{C}$, lower stems of both noninfected and 'Candidatus Liberibacter solanacearum'-infected potato plants. Pairwise comparisons made by follow-up analyses of variance ( $F$ values) are given for each compound and tissue; *, **, and *** indicate $P<0.05,0.01$, and 0.001 , respectively. 
ception was sesquiterpenoid levels in the upper stem. Some of these results could be considered in contrast to previous results of ' $C a$. L. solanacearum'-induced physiological shifts in tuber tissue, which observed increases in the majority of amino acids, sugars, and phenolics in ' $\mathrm{Ca}$. L. solanacearum'-infected plants compared with those noninfected $(33,41,44)$.

Unlike previous studies examining metabolites in potato tubers $(33,41,44)$, the levels of metabolites in this study may have been affected by psyllid feeding in addition to ' $\mathrm{C}$. . L. solanacearum' infection. However, ' $C a$. L. solanacearum' infections would not be possible in field conditions without feeding from psyllids; therefore, such changes in chemistry due to both ' $C a$. L. solanacearum' infection and psyllid infestation would be expected in all ZC-diseased plants. In previous studies, psyllid feeding was shown to affect host chemistry, albeit when much greater numbers of psyllids were allowed to feed on tissues ( 7.5 per versus 20 per plant by Sengoda et al. [37] or even greater insect densities
$[22,32])$ up to when plant samples were collected $(22,32,37)$. Currently, frequent insecticide application is the only way to limit ' $C a$. L. solanacearum' spread (16); therefore, this study tried to simulate a more likely scenario of ' $\mathrm{Ca}$. L. solanacearum' infection, whereby potato plants had psyllids killed by insecticides after a limited feeding period (34). Regardless, based on previous work, psyllids and the psyllid-feeding associated condition known as "psyllid yellows" can cause some foliar symptoms on potato hosts (37). However, such symptoms were never observed to be as severe then when ' $C a$. L. solanacearum' infections also were present (37). Examining specifically the effects of psyllid feeding or ' $C a$. L. solanacearum' infection decoupled from each other were beyond the scope of this work but could provide interesting data and, therefore, should be the focus of future studies.

Regarding the changes in amino acid levels observed by this study, foliar levels of proline and serine were greater in infected plants than controls, whereas foliar levels of asparagine, aspartic

TABLE 3. Mean ( \pm standard error) levels $(\mu \mathrm{g} / \mathrm{g}$ FW) of phenolics in noninfected and infected leaf, upper stem, and lower stem tissue

\begin{tabular}{|c|c|c|c|c|c|}
\hline Tissue, compound & Noninfected & Infected & $F(N=32)$ & Symptom $\rho(N=14-16)$ & Titer $\rho(N=16)$ \\
\hline \multicolumn{6}{|l|}{ Leaf } \\
\hline Betuloside & $43.9 \pm 6.9$ & $23.7 \pm 14.7$ & $6.622 *$ & 0.453 & $-0.662 * *$ \\
\hline Chichoric acid & $25.5 \pm 3.7$ & $18.8 \pm 2.3$ & 2.460 & 0.032 & -0.385 \\
\hline Dichlorogenic acid & $2.1 \pm 0.26$ & $7.7 \pm 0.71$ & $54.747 * * *$ & 0.375 & -0.088 \\
\hline Diflavone & $25.0 \pm 3.4$ & $14.3 \pm 2.8$ & $6.016^{*}$ & 0.199 & $-0.535^{*}$ \\
\hline Epicatechin gallate & $12.8 \pm 1.8$ & $25.3 \pm 4.9$ & $5.707^{*}$ & $0.581^{*}$ & 0.059 \\
\hline Ferulic acid derivative & $41.9 \pm 4.6$ & $21.5 \pm 2.0$ & $16.690 * * *$ & $0.611^{*}$ & $-0.776^{* * *}$ \\
\hline Flavanol methyl ester & $11.1 \pm 3.3$ & $14.2 \pm 3.6$ & 0.413 & $0.586^{*}$ & -0.047 \\
\hline Flavonoid glycoside 1 & $19.2 \pm 2.8$ & $31.0 \pm 5.9$ & 3.236 & 0.503 & 0.241 \\
\hline Flavonoid glycoside 2 & $11.7 \pm 1.8$ & $14.9 \pm 3.4$ & 0.716 & 0.151 & -0.029 \\
\hline Flavonoid glycoside 3 & $17.9 \pm 4.4$ & $11.3 \pm 4.1$ & 1.203 & 0.446 & $-0.594^{*}$ \\
\hline Procyanidin A & $66.6 \pm 10.6$ & $32.9 \pm 7.5$ & $6.784^{*}$ & 0.014 & 0.197 \\
\hline Procyanidin B isomer 1 & $265.0 \pm 38$ & $256.0 \pm 37$ & 0.029 & $0.636^{*}$ & 0.006 \\
\hline Procyanidin B isomer 2 & $50.6 \pm 9.5$ & $49.2 \pm 11.3$ & 0.010 & $0.641^{*}$ & -0.209 \\
\hline Procyanidin B isomer 3 & $39.3 \pm 5.7$ & $54.8 \pm 9.5$ & 1.956 & $0.618 *$ & -0.388 \\
\hline Procyanidin B isomer 4 & $16.7 \pm 2.5$ & $99.1 \pm 5.9$ & $165.368 * * *$ & 0.357 & -0.185 \\
\hline Procyanidin C isomer 1 & $79.6 \pm 12.0$ & $75.6 \pm 10.6$ & 0.063 & $0.576^{*}$ & 0.041 \\
\hline Procyanidin $\mathrm{C}$ isomer 2 & $29.0 \pm 4.1$ & $26.7 \pm 3.0$ & 0.221 & 0.496 & -0.368 \\
\hline Procyanidin digalloyl dimer & $20.6 \pm 2.0$ & $39.8 \pm 7.0$ & $6.950^{*}$ & -0.231 & 0.197 \\
\hline Protocatechuic acid hexoside & $23.0 \pm 2.6$ & $22.4 \pm 2.2$ & 0.037 & -0.483 & -0.032 \\
\hline Quercetin diglycoside 1 & $15.8 \pm 3.4$ & $12.8 \pm 3.1$ & 0.422 & 0.126 & 0.041 \\
\hline Quercetin diglycoside 2 & $29.7 \pm 4.7$ & $17.8 \pm 3.3$ & $4.194 *$ & 0.476 & -0.044 \\
\hline Quinic acid & $25.0 \pm 2.8$ & $7.5 \pm 1.10$ & $34.093 * * *$ & -0.387 & 0.197 \\
\hline \multicolumn{6}{|l|}{ Upper stem } \\
\hline Apigenin glucoside & $8.69 \pm 2.48$ & $7.25 \pm 1.51$ & 0.246 & $0.718 * *$ & -0.206 \\
\hline Chichoric acid & $3.99 \pm 0.78$ & $2.30 \pm 0.37$ & 3.856 & 0.263 & 0.088 \\
\hline Chlorogenic acid & $23.1 \pm 3.67$ & $31.9 \pm 4.2$ & 2.469 & $0.777 * *$ & 0.082 \\
\hline Cryptochlorogenic acid & $96.9 \pm 13.8$ & $90.5 \pm 10.6$ & 0.136 & 0.082 & -0.032 \\
\hline Ellagitannin 1 & $24.8 \pm 6.7$ & $53.0 \pm 0.7$ & $17.52 * * *$ & -0.237 & -0.347 \\
\hline Ellagitannin 2 & $13.5 \pm 2.6$ & $22.0 \pm 0.9$ & $9.475^{* *}$ & -0.144 & -0.188 \\
\hline Flavonoid diglycoside & $3.47 \pm 0.89$ & $1.88 \pm 0.40$ & 2.645 & 0.295 & 0.097 \\
\hline Flavonoid glycoside & $5.44 \pm 0.83$ & $6.18 \pm 1.26$ & 0.238 & 0.240 & 0.153 \\
\hline Flavonoid rhamnoside glycoside & $13.70 \pm 4.1$ & $31.8 \pm 0.5$ & $19.443 * * *$ & -0.100 & 0.015 \\
\hline Protocatechuic acid hexoside & $6.33 \pm 1.94$ & $7.92 \pm 1.23$ & 0.480 & -0.225 & 0.279 \\
\hline Quercetin diglycoside & $2.60 \pm 0.64$ & $2.30 \pm 0.42$ & 0.152 & 0.055 & 0.350 \\
\hline Quinic acid & $4.92 \pm 0.94$ & $8.56 \pm 0.79$ & $8.801 * *$ & -0.318 & 0.224 \\
\hline Rutin & $6.93 \pm 2.7$ & $5.09 \pm 0.95$ & 0.430 & $0.687 * *$ & -0.076 \\
\hline Syringic acid derivative & $1.51 \pm 0.20$ & $1.16 \pm 0.10$ & 2.534 & -0.354 & -0.009 \\
\hline \multicolumn{6}{|l|}{ Lower stem } \\
\hline Apigenin glucoside & $5.47 \pm 1.02$ & $5.23 \pm 1.19$ & 0.022 & 0.338 & 0.091 \\
\hline Chichoric acid & $1.60 \pm 0.30$ & $1.09 \pm 0.176$ & 2.196 & $0.649 * *$ & -0.091 \\
\hline Chlorogenic acid & $18.4 \pm 1.9$ & $28.2 \pm 3.8$ & $5.259 *$ & $0.689 * *$ & -0.000 \\
\hline Cryptochlorogenic acid & $42.7 \pm 4.5$ & $44.4 \pm 4.6$ & 0.071 & $0.546^{*}$ & 0.168 \\
\hline Ellagitannin 1 & $1.50 \pm 0.16$ & $53.8 \pm 0.8$ & $4,075 * * *$ & 0.222 & -0.418 \\
\hline Ellagitannin 2 & $2.80 \pm 0.21$ & $23.7 \pm 0.57$ & $1,187 * * *$ & 0.474 & -0.297 \\
\hline Flavonoid diglycoside & $1.24 \pm 0.19$ & $0.728 \pm 0.248$ & 2.681 & 0.312 & 0.012 \\
\hline Flavonoid glycoside & $2.94 \pm 0.46$ & $4.15 \pm 0.36$ & $4.232^{*}$ & -0.204 & 0.341 \\
\hline Flavonoid rhamnoside glycoside & $1.94 \pm 0.41$ & $34.7 \pm 0.4$ & $1,652 * * *$ & 0.119 & $0.529 *$ \\
\hline Protocatechuic acid hexoside & $5.69 \pm 1.47$ & $7.40 \pm 0.97$ & 0.943 & -0.421 & 0.309 \\
\hline Quercetin diglycoside & $2.07 \pm 0.40$ & $1.71 \pm 0.33$ & 0.502 & 0.392 & -0.035 \\
\hline Quinic acid & $11.7 \pm 1.0$ & $10.9 \pm 0.8$ & 0.409 & -0.061 & $0.624 * *$ \\
\hline Rutin & $6.51 \pm 1.63$ & $7.94 \pm 1.33$ & 0.463 & -0.073 & 0.276 \\
\hline Syringic acid derivative & $0.733 \pm 0.081$ & $0.484 \pm 0.032$ & $8.154 * *$ & 0.371 & -0.162 \\
\hline
\end{tabular}

${ }^{a} F$ test and Spearman's $\rho$ correlation statistics are given; ${ }^{*}, * *$, and $* * *$ indicate $P<0.05,0.01$, and 0.001 , respectively. 
acid, and glutamine were less in infected plants compared with controls (Table 1). These findings are similar to those by Wallis et al. (44), who observed greater proline and serine in tubers from plants infected for 4 weeks, yet reduced levels of asparagine, aspartic acid, and glutamic acid in the same tubers. By contrast, stem levels of asparagine and aspartic acid were greater in ' $\mathrm{Ca}$. L. solanacearum'-infected plants. In foliar tissues, levels of asparagine, aspartic acid, and glutamine being lower in ' $\mathrm{Ca}$. L. solanacearum'-infected than noninfected plants might be attributed to these amino acids acting as precursors to stress-related metabolites, including proline, arginine, or $\gamma$-amino butyric acid (6). These compounds being lowered in foliage of infected plants also might be a result ' $C a$. L. solanacearum' or psyllid consumption, which has been found with another biotrophic pathogen (38), or due to translocation to the stem. The possible translocation to the stem could be a potential explanation of why asparagine and aspartic acid were greater in ' $C a$. L. solanacearum'-infected plants verses controls in the stem but not in foliage. Increased foliar levels of proline in response to ' $C a$. L. solanacearum' infection might serve to protect diseased plants from reactive oxygen species (3) as well as serve in cell wall strengthening via production of proline- and hydroxyproline-rich proteins (9). Thicker cell walls could, hypothetically, be a host defense response to both ' $\mathrm{Ca}$. L. solanacearum' and the potato psyllid vector.

Sugar levels in this study were generally greater in noninfected than 'Ca. L. solanacearum'-infected plants (Fig. 1). Gao et al. (15) also observed greater glucose levels in noninfested plants compared with those likely infected with ' $\mathrm{Ca}$. L. solanacearum' (i.e., those infested with ' $C a$. L. solanacearum'-positive potato psyllids). This is likely because of decreased photosynthesis because ZC symptoms developed due to necrosis of leaf tissues, the use of carbohydrates by the host to provide energy for a defense response, and ' $\mathrm{Ca}$. L. solanacearum' consuming sugars

TABLE 4. Mean ( \pm standard error) levels (ng/g FW) of monoterpenoids in noninfected and infected leaf, upper stem, and lower stem tissue ${ }^{\mathrm{a}}$

\begin{tabular}{|c|c|c|c|c|c|c|c|}
\hline Tissue & $\begin{array}{l}\text { Retention } \\
\text { time }\end{array}$ & Compound & Noninfected & Infected & $\begin{array}{c}F \\
(N=28-32)\end{array}$ & $\begin{array}{l}\text { Symptom } \rho \\
(N=12-16)\end{array}$ & $\begin{array}{c}\text { Titer } \rho \\
(N=14-16)\end{array}$ \\
\hline \multirow[t]{17}{*}{ Leaf } & 12.11 & Camphene $^{\mathrm{b}}$ & $8.98 \pm 0.26$ & $10.7 \pm 0.2$ & $24.811 * * *$ & -0.463 & -0.107 \\
\hline & 12.28 & $\Delta$-2-Carene ${ }^{\mathrm{b}}$ & $9.14 \pm 0.33$ & $10.9 \pm 0.3$ & $16.937 * * *$ & -0.444 & -0.284 \\
\hline & 12.93 & $(+)-\beta$-Pinene & $5.78 \pm 0.74$ & $4.78 \pm 0.78$ & 0.859 & 0.275 & 0.075 \\
\hline & 13.02 & (-)- $\beta$-Pinene & $1.98 \pm 0.15$ & $2.04 \pm 0.17$ & 0.064 & -0.108 & 0.031 \\
\hline & 13.54 & $\alpha$-Phellandrene & $9.92 \pm 0.19$ & $11.2 \pm 0.2$ & $18.500 * * *$ & -0.400 & -0.226 \\
\hline & 13.77 & $\alpha$-Terpinene & $3.17 \pm 0.12$ & $3.80 \pm 0.23$ & $5.931 *$ & -0.437 & 0.042 \\
\hline & 14.67 & $\beta$-Phellandrene ${ }^{b}$ & $18.1 \pm 0.5$ & $22.2 \pm 0.5$ & $34.643 * * *$ & -0.444 & -0.248 \\
\hline & 14.82 & Para-cymene & $17.1 \pm 0.4$ & $20.6 \pm 0.5$ & $29.345 * * *$ & -0.393 & -0.323 \\
\hline & 14.95 & $\beta$-Ocimene & $8.91 \pm 0.21$ & $10.3 \pm 0.2$ & $21.628 * * *$ & -0.451 & -0.196 \\
\hline & 15.22 & $\gamma$-Terpinene & $4.40 \pm 0.13$ & $5.38 \pm 0.11$ & $32.767 * * *$ & -0.429 & -0.218 \\
\hline & 15.34 & 1,8-Cineole & $3.09 \pm 0.10$ & $3.63 \pm 0.11$ & $13.526^{* *}$ & 0.051 & -0.262 \\
\hline & 15.98 & $\alpha$-Terpinolene & $1.56 \pm 0.06$ & $1.67 \pm 0.05$ & 1.987 & -0.160 & 0.033 \\
\hline & 18.00 & Allo-ocimene & $2.11 \pm 0.35$ & $3.28 \pm 0.60$ & 2.880 & -0.240 & -0.244 \\
\hline & 19.01 & $\beta$-Linalool & $8.47 \pm 0.51$ & $10.4 \pm 0.7$ & $4.813 *$ & -0.036 & -0.081 \\
\hline & 19.99 & Bornyl acetate & $11.2 \pm 0.3$ & $12.8 \pm 0.2$ & $19.180 * * *$ & -0.058 & -0.358 \\
\hline & 21.23 & Terpin-4-ol & $24.7 \pm 0.8$ & $30.9 \pm 0.6$ & $37.334 * * *$ & -0.466 & -0.020 \\
\hline & 25.01 & Neryl acetate & $7.25 \pm 0.57$ & $8.60 \pm 0.32$ & $4.224 *$ & -0.298 & -0.468 \\
\hline \multirow[t]{17}{*}{ Upper stem } & 12.11 & Camphene $^{\mathrm{b}}$ & $8.63 \pm 0.22$ & $11.5 \pm 0.2$ & $79.548 * * *$ & 0.055 & 0.222 \\
\hline & 12.28 & $\Delta$-2-Carene ${ }^{\mathrm{b}}$ & $8.46 \pm 0.24$ & $12.0 \pm 0.3$ & $101.485 * * *$ & 0.075 & 0.218 \\
\hline & 12.93 & $(+)$ - $\beta$-Pinene & $2.58 \pm 0.18$ & $3.11 \pm 0.10$ & $6.469 *$ & 0.259 & 0.137 \\
\hline & 13.02 & $(-)-\beta$-Pinene & $2.01 \pm 0.10$ & $2.65 \pm 0.12$ & $17.316^{* * * *}$ & 0.070 & 0.116 \\
\hline & 13.54 & $\alpha$-Phellandrene & $9.49 \pm 0.21$ & $12.1 \pm 0.2$ & $71.796 * * *$ & 0.049 & 0.494 \\
\hline & 13.77 & $\alpha$-Terpinene & $3.18 \pm 0.8$ & $4.63 \pm 0.21$ & $42.090 * * *$ & -0.336 & $0.565^{*}$ \\
\hline & 14.67 & $\beta$-Phellandrene ${ }^{b}$ & $17.4 \pm 0.4$ & $24.2 \pm 0.4$ & $122.005 * * *$ & -0.017 & 0.450 \\
\hline & 14.82 & Para-cymene & $16.4 \pm 0.4$ & $22.4 \pm 4.1$ & $114.829 * * *$ & 0.075 & 0.358 \\
\hline & 14.95 & $\beta$-Ocimene & $8.38 \pm 0.19$ & $10.8 \pm 0.19$ & $80.406^{* * *}$ & 0.054 & 0.440 \\
\hline & 15.22 & $\gamma$-Terpinene & $4.30 \pm 0.13$ & $5.83 \pm 0.13$ & $69.736^{* * * *}$ & 0.024 & 0.396 \\
\hline & 15.34 & 1,8-Cineole & $2.74 \pm 0.10$ & $3.65 \pm 0.08$ & $53.549 * * *$ & -0.235 & 0.462 \\
\hline & 15.98 & $\alpha$-Terpinolene & $1.32 \pm 0.05$ & $1.90 \pm 0.05$ & $72.937 * * *$ & 0.199 & 0.178 \\
\hline & 18.00 & Allo-ocimene & $1.56 \pm 0.42$ & $2.16 \pm 0.58$ & 0.731 & 0.075 & 0.444 \\
\hline & 19.01 & $\beta$-Linalool & $6.00 \pm 0.35$ & $7.36 \pm 0.29$ & $8.759 * *$ & -0.240 & $0.544 *$ \\
\hline & 19.99 & Bornyl acetate & $12.0 \pm 0.4$ & $12.3 \pm 0.3$ & 0.482 & -0.064 & $0.588 *$ \\
\hline & 21.23 & Terpin-4-ol & $29.2 \pm 1.1$ & $31.0 \pm 0.7$ & 1.733 & -0.230 & $0.638 * *$ \\
\hline & 25.01 & Neryl acetate & $6.49 \pm 0.31$ & $6.24 \pm 0.24$ & 0.387 & -0.327 & $0.538 *$ \\
\hline \multirow[t]{17}{*}{ Lower stem } & 12.11 & Camphene ${ }^{b}$ & $8.04 \pm 0.19$ & $10.6 \pm 0.3$ & $62.271 * * *$ & 0.073 & -0.103 \\
\hline & 12.28 & $\Delta$-2-Carene ${ }^{\mathrm{b}}$ & $8.06 \pm 0.17$ & $10.9 \pm 0.25$ & $88.823 * * *$ & 0.162 & 0.043 \\
\hline & 12.93 & $(+)$ - $\beta$-Pinene & $2.39 \pm 0.11$ & $2.82 \pm 0.10$ & $8.451 * *$ & -0.379 & 0.024 \\
\hline & 13.02 & $(-)-\beta$-Pinene & $1.88 \pm 0.11$ & $2.56 \pm 0.09$ & $23.794 * * *$ & 0.074 & -0.296 \\
\hline & 13.54 & $\alpha$-Phellandrene & $9.12 \pm 0.83$ & $11.3 \pm 0.18$ & $60.718 * * *$ & -0.017 & 0.029 \\
\hline & 13.77 & $\alpha$-Terpinene & $2.96 \pm 0.10$ & $4.55 \pm 0.19$ & $50.764 * * *$ & 0.142 & -0.317 \\
\hline & 14.67 & $\beta$-Phellandrene ${ }^{\mathrm{b}}$ & $16.3 \pm 0.4$ & $21.7 \pm 0.5$ & $75.295^{* * *}$ & 0.096 & -0.138 \\
\hline & 14.82 & Para-cymene & $15.2 \pm 0.4$ & $20.4 \pm 0.4$ & $85.378 * * *$ & 0.082 & -0.138 \\
\hline & 14.95 & $\beta$-Ocimene & $7.67 \pm 0.20$ & $9.78 \pm 1.67$ & $65.959 * * *$ & 0.017 & -0.205 \\
\hline & 15.22 & $\gamma$-Terpinene & $3.86 \pm 0.11$ & $5.23 \pm 0.10$ & $90.078 * * *$ & -0.017 & -0.327 \\
\hline & 15.34 & 1,8-Cineole & $2.60 \pm 0.09$ & $3.26 \pm 0.10$ & $23.328 * * *$ & 0.126 & -0.178 \\
\hline & 15.98 & $\alpha$-Terpinolene & $1.39 \pm 0.04$ & $1.77 \pm 0.04$ & $43.954 * * *$ & -0.062 & -0.371 \\
\hline & 18.00 & Allo-ocimene & $1.31 \pm 0.10$ & $1.92 \pm 0.50$ & 1.360 & -0.336 & 0.374 \\
\hline & 19.01 & $\beta$-Linalool & $4.25 \pm 0.22$ & $6.48 \pm 0.18$ & $59.900 * * *$ & -0.113 & 0.122 \\
\hline & 19.99 & Bornyl acetate & $10.1 \pm 0.3$ & $11.7 \pm 0.2$ & $19.551 * * *$ & -0.333 & -0.185 \\
\hline & 21.23 & Terpin-4-ol & $22.6 \pm 1.0$ & $28.3 \pm 0.6$ & $24.033 * * *$ & -0.175 & 0.029 \\
\hline & 25.01 & Neryl acetate & $3.91 \pm 0.29$ & $5.57 \pm 0.25$ & $18.863 * * *$ & -0.425 & 0.035 \\
\hline
\end{tabular}

${ }^{\text {a }} F$ test and Spearman's $\rho$ correlation statistics are given; *, **, and *** indicate $P<0.05,0.01$, and 0.001 , respectively.

b Compounds putatively identified based on relative retention times and mass spectra. 
for energy. Glucose levels also might have been converted to starch in foliage (15), although this study did not measure starch levels to confirm this. These findings are different than observations of ' $C a$. L. solanacearum' infected tubers $(33,44)$. However, in tubers, ' $C a$. L. solanacearum' infection likely causes tuber physiological changes that contribute to the conversion of starch to reducing sugars. This would result in greater levels of individual disaccharides and monosaccharides in tubers of ' $\mathrm{Ca}$. $\mathrm{L}$. solanacearum'-infected plants (44).

Depending on tissue, phenolics involved in cell wall strengthening - in particular, ellagitannins (which are hydrolysable tannins) in stems-were greater in ' $\mathrm{Ca}$. L. solanacearum'-infected than noninfected plants. Therefore, it might be hypothesized that the primary production of phenolics in response to ' $\mathrm{Ca}$. L. solanacearum' infection was targeted around making thicker cell walls to limit pathogen movement and access to resources. Likewise, because the ' $C a$. L. solanacearum' bacterial infection process involved psyllid feeding, the thicker cell walls also could be a hypothesized host defense response against the vectors. However, direct cell wall measurements were not made and, therefore, should be verified in future studies and subsequently correlated with pathogen titers and insect behaviors. This formation of thicker cell walls likely does not occur in tubers in response to 'Ca. L. solanacearum' infection, which is why, in tubers, no decreases in phenolic levels were observed (44). However, levels of most phenolics were not significantly different between ' $\mathrm{Ca}$. L. solanacearum'-infected and noninfected plants. Certain flavonoid and other phenolics, which either increased in response to infection (e.g., flavonoid rhamnoside glycoside in stems) or were positively correlated with symptoms (e.g., certain procyanidins in leaves and chlorogenic acid in stems) (Table 3), could have been increased by the host in an attempt to ameliorate disease stress or resist ' $C a$. L. solanacearum' and its vector (16,19,31). Greater flavonoid and chlorogenic acid levels also were observed in potato tubers in response to ' $C a$. L. solanacearum' infection, likely for similar reasons (44).

Levels of most monoterpenoids were much greater in ' $C a$. L. solanacearum'-infected than noninfected plants, although few significant correlations were observed between monoterpenoid levels with ZC symptoms or ' $C a$. L. solanacearum' titers. Sesquiterpenoid levels in leaves and lower stem tissue also were increased in ' $C a$. L. solanacearum'-infected plants, although upper stem sesquiterpenoid levels did not differ. It is difficult to explain why upper stem sesquiterpenoid levels did not differ between noninfected and infected hosts when the other tissues differed, although some reports have observed differential changes between tissues and parts of the plant before (5) and this observation warrants consideration in future studies. Most previous

TABLE 5. Mean ( \pm standard error) levels (ng/g FW) of sesquiterpenoids in noninfected and infected leaf, upper stem, and lower stem tissue

\begin{tabular}{|c|c|c|c|c|c|c|c|}
\hline Tissue & $\begin{array}{l}\text { Retention } \\
\text { time }\end{array}$ & Compound & Noninfected & Infected & $\begin{array}{c}F \\
(N=28-32)\end{array}$ & $\begin{array}{l}\text { Symptom } \rho \\
(N=12-16)\end{array}$ & $\begin{array}{c}\text { Titer } \rho \\
(N=14-16)\end{array}$ \\
\hline \multirow[t]{14}{*}{ Leaf } & 24.80 & $\alpha$-Copanene & $2.83 \pm 0.22$ & $3.75 \pm 0.09$ & $15.276^{* *}$ & -0.240 & 0.059 \\
\hline & 25.28 & $\alpha$-Gurjunene ${ }^{b}$ & $3.79 \pm 0.24$ & $5.03 \pm 0.09$ & $23.906 * * *$ & -0.393 & 0.007 \\
\hline & 25.56 & $\beta$-Caryophellene & $7.18 \pm 0.31$ & $8.47 \pm 0.16$ & $13.605 * *$ & -0.473 & -0.191 \\
\hline & 25.67 & $\beta$-Gurjunene ${ }^{b}$ & $6.45 \pm 1.26$ & $4.87 \pm 0.67$ & 1.226 & -0.036 & 0.011 \\
\hline & 26.60 & $(Z, E)$ - $\alpha$-farnesene & $4.85 \pm 0.26$ & $5.95 \pm 0.15$ & $13.373 * *$ & -0.467 & 0.396 \\
\hline & 26.70 & $\alpha$-Humulene & $8.53 \pm 0.34$ & $9.43 \pm 0.18$ & $5.567 *$ & -0.378 & -0.314 \\
\hline & 26.85 & $(E, E)$ - $\alpha$-farnesene & $5.17 \pm 0.25$ & $6.42 \pm 0.13$ & $19.835 * * *$ & -0.393 & -0.037 \\
\hline & 27.31 & $(E, E)$ - $\beta$-farnesene & $11.3 \pm 1.3$ & $11.0 \pm 0.6$ & 0.068 & -0.400 & 0.231 \\
\hline & 27.56 & $\alpha$-Muurolene ${ }^{b}$ & $7.37 \pm 0.48$ & $8.48 \pm 0.18$ & $4.673^{*}$ & -0.168 & -0.040 \\
\hline & 28.64 & $\Delta$-Cadinene ${ }^{\mathrm{b}}$ & $3.36 \pm 0.22$ & $3.89 \pm 0.12$ & 4.159 & 0.211 & -0.235 \\
\hline & 30.53 & $\Delta$-Cadinol $^{\mathrm{b}}$ & $5.94 \pm 0.24$ & $6.69 \pm 0.14$ & $7.455^{*}$ & -0.590 & 0.086 \\
\hline & 30.69 & Germacrene $\mathrm{D}^{\mathrm{b}}$ & $15.9 \pm 0.7$ & $17.7 \pm 0.3$ & $5.617^{*}$ & -0.306 & -0.275 \\
\hline & 31.51 & Germacrene D-4-ol ${ }^{\mathrm{b}}$ & $10.4 \pm 0.4$ & $12.0 \pm 0.3$ & $9.598 * *$ & -0.480 & -0.262 \\
\hline & 33.02 & Citronellyl isobutyrate ${ }^{b}$ & $21.0 \pm 0.7$ & $25.8 \pm 0.5$ & $26.879 * * *$ & -0.546 & -0.099 \\
\hline \multirow[t]{14}{*}{ Upper stem } & 24.80 & $\alpha$-Copanene & $3.44 \pm 0.14$ & $3.28 \pm 0.11$ & 0.791 & -0.204 & $0.721 * *$ \\
\hline & 25.28 & $\alpha$-Gurjunene ${ }^{b}$ & $5.54 \pm 0.26$ & $5.22 \pm 0.19$ & 1.008 & -0.230 & $0.594 *$ \\
\hline & 25.56 & $\beta$-Caryophellene & $9.41 \pm 0.42$ & $8.55 \pm 0.28$ & 2.867 & 0.038 & $0.556^{*}$ \\
\hline & 25.67 & $\beta$-Gurjunene ${ }^{b}$ & $0.754 \pm 0.034$ & $0.782 \pm 0.044$ & 0.244 & -0.415 & 0.292 \\
\hline & 26.60 & $(Z, E)$ - $\alpha$-farnesene & $5.52 \pm 0.20$ & $5.12 \pm 0.13$ & 2.770 & -0.156 & $0.662 * *$ \\
\hline & 26.70 & $\alpha$-Humulene & $9.52 \pm 0.34$ & $8.88 \pm 0.21$ & 2.579 & -0.131 & $0.634 * *$ \\
\hline & 26.85 & $(E, E)$ - $\alpha$-farnesene & $6.33 \pm 0.25$ & $5.94 \pm 0.17$ & 1.689 & -0.102 & $0.726 * *$ \\
\hline & 27.31 & $(E, E)$ - $\beta$-farnesene & $8.35 \pm 0.33$ & $7.80 \pm 0.23$ & 1.869 & -0.073 & $0.597 *$ \\
\hline & 27.56 & $\alpha$-Muurolene ${ }^{b}$ & $7.87 \pm 0.34$ & $7.59 \pm 0.23$ & 0.473 & -0.283 & $0.647 * *$ \\
\hline & 28.64 & $\Delta$-Cadinene $\mathrm{e}^{\mathrm{b}}$ & $3.54 \pm 0.14$ & $3.57 \pm 0.07$ & 0.021 & -0.234 & $0.552 *$ \\
\hline & 30.53 & $\Delta$-Cadinol $^{\mathrm{b}}$ & $6.24 \pm 0.24$ & $4.92 \pm 0.12$ & $23.092 * * *$ & 0.138 & $0.594 *$ \\
\hline & 30.69 & Germacrene $\mathrm{D}^{\mathrm{b}}$ & $19.1 \pm 0.8$ & $18.7 \pm 0.4$ & 0.190 & -0.362 & $0.629 * *$ \\
\hline & 31.51 & Germacrene D-4-ol ${ }^{\mathrm{b}}$ & $10.9 \pm 0.3$ & $9.89 \pm 0.24$ & $5.860 *$ & 0.078 & $0.630 * *$ \\
\hline & 33.02 & Citronellyl isobutyrate $^{\mathrm{b}}$ & $23.8 \pm 0.8$ & $23.6 \pm 0.6$ & 0.022 & 0.005 & $0.606 *$ \\
\hline \multirow[t]{14}{*}{ Lower stem } & 24.80 & $\alpha$-Copanene & $2.17 \pm 0.17$ & $3.06 \pm 0.10$ & $19.731 * * *$ & -0.456 & -0.088 \\
\hline & 25.28 & $\alpha$-Gurjunene ${ }^{b}$ & $3.68 \pm 0.25$ & $4.71 \pm 0.14$ & $13.727 * *$ & -0.339 & 0.003 \\
\hline & 25.56 & $\beta$-Caryophellene & $6.80 \pm 0.31$ & $7.72 \pm 0.20$ & $6.430 *$ & 0.017 & 0.091 \\
\hline & 25.67 & $\beta$-Gurjunene ${ }^{b}$ & $0.604 \pm 0.035$ & $0.705 \pm 0.046$ & 2.978 & $-0.724 * *$ & 0.191 \\
\hline & 26.60 & $(Z, E)$ - $\alpha$-farnesene & $3.83 \pm 0.23$ & $4.88 \pm 0.11$ & $18.044 * * *$ & $-0.627 * *$ & 0.187 \\
\hline & 26.70 & $\alpha$-Humulene & $7.35 \pm 0.33$ & $8.22 \pm 0.18$ & $5.695^{*}$ & $-0.539 *$ & -0.102 \\
\hline & 26.85 & $(E, E)$ - $\alpha$-farnesene & $4.43 \pm 0.28$ & $5.59 \pm 0.14$ & $14.037 * *$ & $-0.535^{*}$ & 0.065 \\
\hline & 27.31 & $(E, E)$ - $\beta$-farnesene & $5.84 \pm 0.34$ & $7.09 \pm 0.20$ & $10.341 * *$ & -0.470 & 0.091 \\
\hline & 27.56 & $\alpha$-Muurolene ${ }^{\mathrm{b}}$ & $5.64 \pm 0.41$ & $7.21 \pm 0.22$ & $11.658 * *$ & $-0.748 * *$ & 0.109 \\
\hline & 28.64 & $\Delta$-Cadinene ${ }^{\mathrm{b}}$ & $2.50 \pm 0.15$ & $3.09 \pm 0.06$ & $14.363 * *$ & -0.387 & -0.102 \\
\hline & 30.53 & $\Delta$-Cadinol $^{\mathrm{b}}$ & $4.04 \pm 0.17$ & $4.51 \pm 0.11$ & $5.659 *$ & $-0.717 * *$ & -0.057 \\
\hline & 30.69 & Germacrene $\mathrm{D}^{\mathrm{b}}$ & $13.3 \pm 0.6$ & $16.0 \pm 0.4$ & $16.734 * * *$ & -0.304 & -0.243 \\
\hline & 31.51 & Germacrene D-4-ol ${ }^{\mathrm{b}}$ & $8.44 \pm 0.41$ & $9.65 \pm 0.26$ & $6.575^{*}$ & $-0.658 * *$ & -0.026 \\
\hline & 33.02 & Citronellyl isobutyrate ${ }^{b}$ & $17.8 \pm 0.8$ & $21.7 \pm 0.5$ & $16.843 * * *$ & $-0.522 *$ & -0.015 \\
\hline
\end{tabular}

${ }^{\text {a }} F$ test and Spearman's $\rho$ correlation statistics are given; *, **, and *** indicate $P<0.05,0.01$, and 0.001 , respectively.

${ }^{\mathrm{b}}$ Compounds putatively identified based on relative retention times and mass spectra. 
studies observing induction of terpenoid production following infection, or the ability of terpenoids to limit pathogen growth, have focused on necrotrophic fungi $(5,16,20,43)$. However, it was observed that Pseudomonas syringae infection can induce terpenoids in Arabidopsis (2), and likely ' $C a$. L. solanacearum' infection of potato plants may increase terpenoid levels as well (13).

Although the role terpenoids have in defense against pathogens remains poorly understood, terpenoids can greatly affect insect behaviors and physiology (20), and levels in the host may have shifted in response to psyllid feeding in combination with ' $\mathrm{Ca}$. L. solanacearum' infection. In fact, in many plants, including potato, it should be expected that psyllid feeding could affect terpenoid levels, perhaps as a specific response to herbivory $(22,32)$. Because ' $\mathrm{Ca}$. L. solanacearum' is vectored by psyllids, the increase in terpenoids as a host response to infection or even prior feeding may alter efficiency of pathogen spread. There is some evidence that increased terpenoids in plant hosts will act as feeding deterrents and increase insect pest movement $(4,12)$. This might increase ' $\mathrm{Ca}$. L. solanacearum' spread by psyllids as long as the bacteria could be acquired prior to insect movement. However, Davis et al. (13) observed that psyllids settled more on plants possibly inoculated with ' $\mathrm{Ca}$. L. solanacearum' 7 days previously than noninoculated controls, even though psyllid-infested plants had greater terpenoid levels. Greater time spent on a plant may reduce spread of ' $\mathrm{Ca}$. L. solanacearum' by lowering plant-to-plant vector movement but it also increases likelihood of ' $\mathrm{Ca}$. L. solanacearum' acquisition by psyllids. Therefore, studies to examine plant-psyllid interactions, especially the influence that terpenoids have on insect behaviors and how that may affect pathogen spread, are warranted because our current knowledge remains limited.

In conclusion, this study observed potential changes in potato physiology in response to ' $\mathrm{Ca}$. L. solanacearum' infection and associated psyllid feeding that could be directly or indirectly associated with the expression of aboveground ZC symptoms in potato plants. These changes in physiology could affect the formation of tubers, because disruptions in metabolism overall should affect resources needed for proper tuber development. Shifts in metabolism in the aboveground portions of potato plants are hypothesized to affect both ' $C a$. L. solanacearum' and its psyllid vector. This, is turn, would affect both spread and progression of $\mathrm{ZC}$ disease in its potato hosts. These results also highlight the need to conduct further studies to examine what occurs when potato plants are attacked by both ' $\mathrm{Ca}$. L. solanacearum' and its psyllid vectors. In particular, future studies are warranted to examine how potato host chemistry is affected by ' $C a$. L. solanacearum'-negative psyllid feeding. Conversely, it would be important to examine how potato host chemistry can be affected by ' $C a$. L. solanacearum' infections that began without psyllid feeding, such as those by graft inoculations. Finally, analyzing changes in defense-related protein levels and regulations of host genes in addition to shifts in host physiology over the course of an infection would aid in developing a very comprehensive picture of potato host responses to ' $\mathrm{Ca}$. L. solanacearum' or psyllids.

\section{ACKNOWLEDGMENTS}

We thank M. Henning, G. Aguilar, A. Fite, N. Goodell, A. Wallingford, and G. Phillips for their technical help in this work; and field technician J. Gray and our undergraduate assistants for their help with fieldwork. This research was funded by the United States Department of Agriculture Specialty Crop Research Initiative grant number 2009-51181-20176 and associated Zebra Chip SCRI/Industry-Funded Mini-Grant. Mention of trade names or commercial products in this publication is solely for the purpose of providing specific information and does not imply recommendation or endorsement by the United States Department of Agriculture (USDA). USDA is an equal opportunity provider and employer.

\section{LITERATURE CITED}

1. Alvarado, V. Y., Odokonyero, D., Duncan, O., Mirkov, T. E., and Scholthof, H. B. 2012. Molecular and physiological properties associated with zebra chip complex disease in potatoes and its relation with Candidatus Liberibacter contents in psyllid vectors. PLoS One 7:e37345.

2. Attaran, E., Rostas, M., and Zeier, J. 2008. Pseudomonas syringae elicits emission of the terpenoid $(E, E)-4,8,12$-trimethyl-1, 3, 7, 11-tridecatetraene in Arabidopsis leaves via jasmonate signaling and expression of the terpene synthase TPS4. Mol. Plant-Microbe Interact. 21:14821497.

3. Banu, N. A., Hoque, A., Watanabe-Sugimoto, M., Matsuoka, K., Nakamura, Y., Shimoishi, Y., and Murata, Y. 2009. Proline and glycinebetaine induce antioxidant defense gene expression and suppress cell death in cultured tobacco cells under salt stress. J. Plant Physiol. 166:146156.

4. Bleeker P.M., Diergaarde, P. J., Ament, K., Guerra, J., Weidner, M., Schutz, S., de Both, M. T. J., Haring, M. A., and Schuurink, R. C. 2009. The role of specific tomato volatiles in tomato-whitefly interaction. Plant Physiol. 151:925-935.

5. Bonello, P., Capretti, P., Luchi, N., Martini, V., and Michelozzi, M. 2008. Systemic effects of Heterobasidion annosum s.s. infection on severity of Diplodia pinea tip blight and terpenoid metabolism in Italian stone pine (Pinus pinea). Tree Physiol. 28:1653-1660.

6. Brauc, S., De Vooght, E., Claeys, M., Höfte, M., and Angenon, G. 2011. Influence of over-expression of cytosolic aspartate aminotransferase on amino acid metabolism and defence responses against Botrytis cinerea infection in Arabidopsis thaliana. J. Plant Physiol. 168:1813-1819.

7. Buchman, J. L., Fisher, T. W., Sengoda, V. G., and Munyaneza, J. E. 2012. Zebra chip progression: From inoculation of potato plants with Liberibacter to development of disease symptoms in tubers. Am. J. Pot. Res. 89:159-168.

8. Buchman, J. L., Heilman, B. E., and Munyaneza, J. E. 2011. Effects of Bactericera cockerelli (Hemiptera: Triozidae) density on zebra chip potato disease incidence, potato yield, and tuber processing quality. J. Econ. Entomol. 104:1783-1792.

9. Cassab, G. I. 1998. Plant cell wall proteins. Annu. Rev. Plant Physiol. Plant Mol. Biol. 49:281-309.

10. Crosslin, J. M., Hamm, P. B., Eggers, J. E., Rondon, S. I., Sengoda, V. G., and Munyaneza, J. E. 2012. First report of zebra chip disease and 'Candidatus Liberibacter solanacearum' on potatoes in Oregon and Washington State. Plant Dis. 96:452.

11. Crosslin, J. M., Olsen, N., and Nolte, P. 2012. First report of zebra chip disease and 'Candidatus Liberibacter solanacearum' on potatoes in Idaho. Plant Dis. 96:453.

12. Dancewicz, K., Gabrys, B., Dams, I., and Wawrzenczyk, C. 2008. Enantiospecific effect of pulegone and pulegone-derived lactones on Myzus persicae (Sulz.) settling and feeding. J. Chem. Ecol. 34:530-538.

13. Davis, T. S., Horton, D. R., Munyaneza, J. E., and Landolt, P. J. 2012. Experimental infection of plants with an herbivore-associated bacterial endosymbiont influences herbivore host selection behavior. PLoS One 7:e49330.

14. Friedman, M., and Levin, C. E. 2008. Review of methods for the reduction of dietary content and toxicity of acrylamide. J. Agric. Food Chem. 56:6113-6140.

15. Gao, F., Jifon, J., Yang, X., and Liu, T.-X. 2009. Zebra chip disease incidence on potato is influenced by timing of potato psyllid infestation, but not by the host plants on which they were reared. Insect Sci. 16:399408.

16. Guenthner, J., Goolsby, J., and Greenway, G. 2012. Use and cost of insecticides to control potato psyllids and zebra chip on potatoes. Southwest. Entomol. 37:263-270.

17. Henriquez, M. A., Adam, L. R., and Daayf, F. 2012. Alteration of secondary metabolites' profile in potato leaves in response to weakly and highly aggressive isolates of Phytophthora infestans. Plant Physiol. Biochem. 57:8-14

18. Janse, J. D. 2012. Bacterial diseases that may or do emerge, with (possible) economic damage for Europe and the Mediterranean basin: Notes on epidemiology, risks, prevention and management on first occurrence. J. Plant Pathol. 94:S5-S29.

19. Kahkonen, M. P., Hopia, A., Vurolela, H. J., Rauha, J. P., Pihlaja, K., Kujala, T. S., and Heinonen, M. 1999. Antioxidant activity of plant extracts containing phenolic compounds. J. Agric. Food Chem. 47:39543962.

20. Keeling, C. I., and Bohlmann, J. 2006. Genes, enzymes and chemicals of terpenoid diversity in the constitutive and induced defence of conifers against insects and pathogens. New Phytol. 170:657-675.

21. Liefting, L. W., Perez-Egusquiza, Z. C., Clover, G. R. G., and Anderson, J. A. D. 2008. A new 'Candidatus Liberibacter' species in Solanum tuberosum in New Zealand. Plant Dis. 92:1474. 
22. Malik, N. S. A., Perez, J. L., Kunta, M., Patt, J. M., and Mangan, R. L. 2013. Changes in free amino acids and polyamine levels in Satsuma leaves in response to Asian citrus psyllid infestation and water stress. Insect Sci. doi:10.1111/1744-7917.12075

23. Mayer, A. M. 2006. Polyphenol oxidases in plants and fungi: Going places? A review. Phytochemistry 67:2318-2331.

24. Miles, G. P., Buchman, J. L., and Munyaneza, J. E. 2009. Impact of zebra chip disease on the mineral content of potato tubers. Am. J. Pot. Res. 86:481-489.

25. Miles, G. P., Samuel, M. A., Chen, J., Civerolo, E. L., and Munyaneza, J. E. 2010. Evidence that cell death is associated with zebra chip disease in potato tubers. Am. J. Pot. Res. 87:337-349.

26. Moreno, J. I., Martin, R., and Castresana, C. 2005. Arabidopsis SHMT1, a serine hydroxymethyltransferase that functions in the photorespiratory pathway influences resistance to biotic and abiotic stress. Plant J. 41:451463.

27. Munyaneza, J. E. 2012. Zebra chip disease of potato: Biology, epidemiology, and management. Am. J. Pot. Res. 89:329-350.

28. Munyaneza, J. E., Buchman, J. L., Sengoda, V. G., Fisher, T. W., and Pearson, C. C. 2011. Susceptibility of selected potato varieties to zebra chip potato disease. Am. J. Pot. Res. 88:435-440.

29. Munyaneza, J. E., Crosslin, J. M., and Upton, J. E. 2007. Association of Bactericera cockerelli (Homoptera: Psyllidae) with "zebra chip", a new potato disease in southwestern United States and Mexico. J. Econ. Entomol. 100:656-663.

30. Navarre, D. A., Shakya, R., Holden, J., and Crosslin, J. M. 2009. LC-MS analysis of phenolic compounds in tubers showing zebra chip symptoms. Am. J. Pot. Res. 86:88-95.

31. Ngadze, E., Icishahayo, D., Coutinho, T. A., and van der Waals, J. E. 2012. Role of polyphenol oxidase, peroxidase, phenylalanine ammonia lyase, chlorogenic acid, and total soluble phenols in resistance of potatoes to soft rot. Plant Dis. 96:186-192.

32. Nissinen, A. I., Lemmetty, A., Pihlava, J. M., Jauhiainen, L., Munyaneza, J. E., and Vanhala, P. 2012. Effects of carrot psyllid (Trioza apicalis) feeding on carrot yield and content of sugars and phenolic compounds. Ann. Appl. Biol. 161:68-80.

33. Rashed, A., Wallis, C. M., Paetzold, L., Workneh, F., and Rush, C. M. 2013. Zebra chip disease and potato biochemistry: Tuber physiological changes in response to 'Candidatus Liberibacter solanacearum' infection over time. Phytopathology 103:419-426.

34. Rashed, A., Workneh, F., Paetzold, L., Gray, J., and Rush, C. M. 2014. Zebra chip disease development in relation to plant age and time of 'Candidatus Liberibacter solanacearum' infection. Plant Dis. 98:24-31.

35. Secor, G. A., and Rivera-Varas, V. 2004. Emerging diseases of cultivated potato and their impact on Latin America. Rev. Latinoam. Papa (Supl.) $1: 1-8$.

36. Secor, G. A., Rivera-Varas, V., Abad, J. A., Lee, I. M., Clover, G. R. G., Liefting, L. W., Li, X., and De Boer, S. H. 2009. Association of 'Candidatus Liberibacter solanacearum' with zebra chip disease of potato established by graft and psyllid transmission, electron microscopy, and PCR. Plant Dis. 93:574-583.

37. Sengoda, V. G., Munyaneza, J. E., Crosslin, J. M., Buchman, J. L., and Pappu, H. R. 2010. Phenotypic and etiological differences between psyllid yellows and zebra chip diseases of potato. Am. J. Pot. Res. 87:41-49.

38. Solomon, P. S., Tan, K. C., and Oliver, R. P. 2003. The nutrient supply of pathogenic fungi: A fertile field for study. Mol. Plant Pathol. 4:203-210.

39. Stadler, R. H., Blank, I., Varga, N., Robert, F., Hau, J., Guy, P. A., Robert, M.-C., and Riediker, S. 2002. Acrylamide from Maillard reaction products. Nature 419:449-450.

40. Swisher, K. D., Munyaneza, J. E., and Crosslin, J. M. 2012. High resolution melting analysis of the cytochrome oxidase I gene identifies three haplotypes of the potato psyllid in the United States. Environ. Entomol. 41:1019-1028.

41. Wallis, C. M., Chen, J., and Civerolo, E. L. 2012. Zebra chip-diseased potato tubers are characterized by increased levels of host phenolics, amino acids, and defense-related proteins. Physiol. Mol. Plant Pathol. 78:66-72.

42. Wallis, C., Eyles, A., McSpadden Gardener, B., Hansen, R., Cipollini, D., Herms, D. A., and Bonello, P. 2008. Systemic induction of phloem secondary metabolism and its relationship to resistance to a canker pathogen in Austrian pine. New Phytol. 177:767-778.

43. Wallis, C. M., Huber, D. P. W., and Lewis, K. J. 2011. Ecosystem, location, and climate effects on foliar secondary metabolites of lodgepole pine populations form central British Columbia. J. Chem. Ecol. 37:607621.

44. Wallis, C. M., Rashed, A., Wallingford, A., Paetzold, L., Workneh, F., and Rush, C. M. 2014. Similarities and differences in physiological response to 'Candidatus Liberibacter solanacearum' infection among different potato cultivars. Phytopathology 104:126-133.

45. Wen A., Lin, H., and Gudmestad, N. C. 2012. Development of PCR assay using SSR primers for detection and genotyping of 'Candidatus Liberibacter solanacearum'. Pages 74-78 in: Proc. 11th Annu. SCRI Zebra Chip Rep. Session. F. Workneh, A. Rashed, and C. M. Rush, eds. Fredric Printing, Aurora, CO.

46. Wen, A., Mallik, I., Alvarado, V. Y., Pasche, J. S., Wang, X., Li, W., Levy, L., Lin, H., Scholthof, H. B., Mirkov, T. E., Rush, C. M., and Gudmestad, N. C. 2009. Detection, distribution, and genetic variability of 'Candidatus Liberibacter' species associated with zebra complex disease of potato in North America. Plant Dis. 93:1102-1115. 